\title{
Squeezing Properties of Measurement Phase Operator in the Superposition of Coherent State and Squeezed State
}

\author{
Yongxin Zhan \\ Department of physics of Sichuan Agriculture University \\ Ya'an 625014, Sichuan, China \\ Tel: 86-835-288-5166_E-mail: zyx073515@163.com
}

\begin{abstract}
The squeezing properties in the superposition of coherent States and squeezed state are investigated by means of the measurement phase operator introduced by Barnett and S M and Pagg D T.
\end{abstract}

Keywords: Superposition of coherent state and squeezed state, Measurement operator, Squeezing

\section{Introduction}

The study of measurement phase operator has given rise to a great many interest recently and attained an important position in quantum optics. As is well known, the amplitude of field in the quantum optics is directly proportional to square root of optic-quantum number operator and the phase of field is described as operator $\exp ( \pm i \varphi)$ by Susskind-Glogower. However the SG phase operators aren't possessed of unitary, hence hermit operators can not be constructed by them. Though two hermit operators

$$
\begin{aligned}
& \cos \varphi=\frac{1}{2}[\exp (i \varphi)+\exp (-i \varphi)] \\
& \sin \varphi=\frac{1}{2}[\exp (i \varphi)-\exp (-i \varphi)]
\end{aligned}
$$

are composed by SG operators, they have not classical property because $<\cos ^{2} \varphi>+<\sin ^{2} \varphi>\neq 1$. On purpose to overcome the difficulty, unitary exponential phase operator and measurement phase operator have been defined by Pegg and Branett in optical field, and their essence is studied in progress (PEGG D T, 1989; BARNETT S M, 1986). In some laboratories, the measurement phase operator usually corresponds to the phase measurement; therefore the measurement phase operator has raised extensive concerns (BARNETT S M, 1986).

In the quantum optics, coherent state and squeezed state are two very important states in the quantum optics (LYNCH R, 1987; WALL D F, 1983). Coherent state is introduced by Schrödinger at first as most classical state in pure quantum state, which is eigenstate of annihilation operator $\hat{a}$, whose two variances of orthogonal amplitude of vibration are equal and they satisfy with minimal uncertainty relation. Squeezed state is squeezing transition vacuum state, whose one orthogonal amplitude variance is less than coherent state; however the other is more than coherent state. Squeezed state has very important application in interference measurement in high degree of accuracy, photo-communication as well as detection of gravitation wave and microwave signal (LOUDON R, 1987; WALL D F, 1983). According to superposition principle, a great many of new quantum states are constructed through superposition of arbitrary state and many favorable works have done. But these superposition states are usually composed of the same sort state, such as superposition of odd-even coherent state or squeezed odd-even coherent state. Some of their non-classical properties on measurement operator have discussed in detail. Recently the new superposition state composed of coherent state and squeezed state is studied and its quantum effect is obtained. In the paper, the squeezed properties of the superposition state on measurement operator are researched by mean of method in the paper (LOUDON R, 1987).

\section{The superposition of coherent state and squeezing state}

The superposition of coherent state and squeezing state is defined

$$
\mid \phi>=N\left(\left|\alpha>+e^{i \varphi}\right| \beta>_{g}\right)
$$


where $\mid \alpha>$ is the coherent state and $\left|\beta>_{g}=S(\xi)\right| m \alpha>$ is squeezed state. $S(\xi)=\exp \left[\frac{1}{2} \xi^{*} \hat{a}^{2}-\frac{1}{2} \xi\left(\hat{a}^{+}\right)^{2}\right]$ is squeezing operator, $\xi=r e^{i \theta}(r \geq 0,0<\theta<2 \pi), r$ is squeezing factor, $\theta$ is squeezing angle. $m=1$ and $m=-1$ denote phase difference between vector states with phase 0 and $\pi$ respectively. $N$ is normal coefficient. To not lose generality, assume that $\alpha$ and $m=1$ is real, $\theta=0$.

According to normal condition, The parameters of $\varphi 、 r 、 \alpha 、 N$ as well as $m$ satisfy the equation

$$
2 N^{2}\left[1+\frac{\cos \varphi}{\cosh ^{1 / 2} r} \exp \left(\frac{m-\cosh r}{\cosh r} \alpha^{2}\right)\right]=1
$$

\section{Orthogonal measurement phase operator}

Pegg and Barnnet have defined orthogonal measurement phase operators

$$
\cos _{M} \varphi=\lambda X_{1}, \sin _{M} \varphi=\lambda X_{2}
$$

where $X_{1}$ and $X_{2}$ are two orthogonal component of optics field

$$
X_{1}=\frac{1}{2}\left(\hat{a}+\hat{a}^{+}\right), X_{2}=\frac{1}{2 i}\left(\hat{a}-\hat{a}^{+}\right)
$$

According to the requirement ofclassical condition: $\left\langle\cos _{M}^{2} \varphi>+<\sin _{M}^{2} \varphi>=1, \lambda\right.$ is $\operatorname{chosen}(\bar{n}+1 / 2)^{-1 / 2}$, where $\bar{n}$ is an average photon number, i.e. $\bar{n}=\left\langle\hat{a}^{+} \hat{a}\right\rangle=\langle N\rangle$.

The below expression is derived From (3)

$$
\bar{n}=N^{2}\left[\alpha^{2}(1+\cosh 2 r-\sinh 2 r)+\sinh ^{2} r+\frac{2 \alpha^{2}(m-\sinh r) \cos \varphi}{(\cosh r)^{3 / 2}} \exp \left(\frac{m-\cosh r}{\cosh r} \alpha^{2}\right)\right]
$$

By means of the relation of measurement phase operator, measurement phase operator and optical number operator, the important equations are obtained

$$
\begin{gathered}
{\left[\cos _{M} \varphi, \sin _{M} \varphi\right]=\frac{i}{2} \lambda^{2},} \\
{\left[\cos _{M} \varphi, N\right]=i \sin _{M} \varphi,} \\
{\left[\sin _{M} \varphi, N\right]=-i \cos _{M} \varphi .}
\end{gathered}
$$

\section{Squeezing effect of measurement phase operator}

In quantum-optical field, when two operators $Y_{1}$ and $Y_{2}$ don't satisfy reciprocal relation, the accuracy of measurement is restricted by measurement indeterminacy principle

$$
<\left(\Delta Y_{1}\right)^{2}><\left(\Delta Y_{2}\right)^{2}>>\frac{1}{4}\left|\left[Y_{1}, Y_{2}\right]\right|^{2}
$$

If the inequality is right

$$
<\left(\Delta Y_{i}\right)^{2}><\frac{1}{2}\left|\left[Y_{1}, Y_{2}\right]\right| \quad(i=1,2)
$$

then there is squeeze effect in component $Y_{i}$ of optical field. With the view of description degree of squeeze, squeezed degree $S_{i}$ is defined 


$$
S_{i}=<\left(\Delta Y_{1}\right)^{2}>-\frac{1}{2}\left|\left[Y_{1}, Y_{2}\right]\right| \quad(i=1,2)
$$

When $S_{i}<0$, it indicated that there is squeezing effect in the $Y_{i}$.

From (8) to (13), the squeezing degrees of measurement phase operator are

$$
\begin{gathered}
S_{1}^{c s}=\frac{\lambda^{2}}{4}\left[2<\hat{a}^{+} \hat{a}>+<\hat{a}^{2}+\hat{a}^{+2}>-<\hat{a}+\hat{a}^{+}>^{2}\right] \\
S_{2}^{c s}=\frac{\lambda^{2}}{4}\left[2<\hat{a}^{+} \hat{a}>-<\hat{a}^{2}+\hat{a}^{+2}>+<\hat{a}-\hat{a}^{+}>^{2}\right] \\
S^{c n}=\frac{\lambda^{2}}{4}\left[2<\hat{a}^{+} \hat{a}>+<\hat{a}^{2}+\hat{a}^{+2}>-<\hat{a}+\hat{a}^{+}>^{2}+1\right]-\frac{|\lambda|}{4}\left|<\hat{a}-\hat{a}^{+}>\right| \\
S^{s n}=\frac{\lambda^{2}}{4}\left[2<\hat{a}^{+} \hat{a}>-<\hat{a}^{2}+\hat{a}^{+2}>+<\hat{a}-\hat{a}^{+}>^{2}+1\right]-\frac{|\lambda|}{4}\left|<\hat{a}+\hat{a}^{+}>\right|
\end{gathered}
$$

Using the expression of coherent state and squeezed state in Fock space

$$
\begin{gathered}
\left|\alpha>=\exp \left(-\frac{|\alpha|^{2}}{2}\right) \sum_{n=0}^{\infty} \frac{\alpha^{n}}{\sqrt{n !}}\right| n> \\
\left|\beta>_{g}=\sum_{n=0}^{\infty}(n ! u)^{-\frac{1}{2}}\left(\frac{v}{2 u}\right)^{\frac{n}{2}} \exp \left(-\frac{|\beta|^{2}}{2}+\frac{v^{*}}{2 u} \beta^{2}\right) H_{n}\left(\frac{\beta}{\sqrt{2 u v}}\right)\right| n>
\end{gathered}
$$

and properties of hermite polynomial

$$
\begin{gathered}
\exp \left(2 x t-t^{2}\right)=\sum_{n=0}^{\infty} \frac{H_{n}(x)}{n !} t^{n} \\
H_{n+1}(x)=2 x H_{n}(x)-2 t H_{n-1}(x)
\end{gathered}
$$

the expressions below are obtained

$$
\begin{aligned}
& <\phi\left|\left(\hat{a}^{2}+\hat{a}^{+2}\right)\right| \phi>=2 N^{2}\left\{\alpha^{2}(1+\cosh 2 r-\sinh 2 r)-\cosh r \sinh r\right. \\
& \left.+\left[\frac{\alpha^{2}}{(\cosh r)^{1 / 2}}+\frac{\alpha^{2}}{(\cosh r)^{5 / 2}}(m-\sinh r)^{2}-\frac{\tanh r}{(\cosh r)^{1 / 2}}\right] \cdot \cos \varphi \exp \left(\frac{m-\cosh r}{\cosh r} \alpha^{2}\right)\right\} \\
& <\phi\left|\left(\hat{a}+\hat{a}^{+}\right)\right| \phi>^{2}=4 \alpha^{2} N^{4}\left[(1+m \cosh r-m \sinh r)^{2}\right. \\
& +\frac{(m+\cosh r-\sinh r)^{2}}{(\cosh r)^{3}} \cos ^{2} \varphi \exp \left(2 \frac{m-\cosh r}{\cosh r} \alpha^{2}\right) \\
& \left.+\frac{2(1+m \cosh r-m \sinh r)(m+\cosh r-\sinh r)}{(\cosh r)^{3 / 2}} \cdot \cos \varphi \exp \left(\frac{m-\cosh r}{\cosh r} \alpha^{2}\right)\right] \\
& \left(<\phi\left|\left(\hat{a}-\hat{a}^{+}\right)\right| \phi>\right)^{2}=-4 \alpha^{2} N^{4} \sin ^{2} \varphi \frac{(\cosh r+\sinh r-m)^{2}}{(\cosh r)^{3}} \exp \left(2 \frac{m-\cosh r}{\cosh r} \alpha^{2}\right) \\
& <\phi\left|\left(\hat{a}+\hat{a}^{+}\right)\right| \phi>=2 \alpha N^{2}[(1+m \cosh r-m \sinh r)+ \\
& \left.\quad \frac{(m+\cosh r-\sinh r)}{(\cosh r)^{3 / 2}} \cos \varphi \exp \left(\frac{m-\cosh r}{\cosh r} \alpha^{2}\right)\right] \\
& <\phi\left|\left(\hat{a}-\hat{a}^{+}\right)\right| \phi>=-2 i \alpha N^{2} \sin \varphi \frac{\cosh r+\sinh r-m}{(\cosh r)^{3 / 2}} \exp \left(\frac{m-\cosh r}{\cosh r} \alpha^{2}\right)
\end{aligned}
$$

To research squeezing properties of measurement operator in (3), the expression (22) (26) are put into (14) (17). By means of numerical calculation technique, some of figures which indicate squeezing degree $S_{i}^{c s}(i=1,2)$ 
$S^{c n}$ and $S^{s n}$ varying with $\alpha, \varphi 、 r$ and $m$ are given. Since curves are symmetric about longitudinal axis, the below curves are given under $\alpha \geq 0$. If $S_{i}^{c s}(i=1,2)$ is negative, there is a kind of squeezing effect of $c s$. If $S^{c n}$ or $S^{s n}$ is negative, there is a kind of squeezing effect of $c n$ or $s n$.

$<$ Figure 1>

In the fig 1, figures from (a) to (c) are drown under $m>0$. Figures from (e) to (f) are drown under $m<0$. They show squeeze degree curves $S_{1}^{c s}$ varying with $\alpha$ under given $r, \varphi$ and $m$. We find that $S_{1}^{c s}$ appears negative in these figures and that squeezed degree increase accompanying with $m$ ascending under $m>0$ and $m$ descending under $m<0$. Hence, there is a kind of squeezing effect of $C S$ in $Y_{1}$ under given parameters.

$<$ Figure 2>

In the fig 2 , figures from (a) to (c) are drown under $m>0$. Figures from (e) to (f) are drown under $m<0$. They show squeeze degree curves $S_{2}^{c s}$ varying with $\alpha$ under give $r, \varphi$ and $m$. We find that $S_{2}^{c s}$ appears negative when $\varphi=0$ or $0.5 \pi$ and positive when $\varphi=\pi$ Hence, there is a kind of squeezing effect of $C S$ in $Y_{2}$ when $\varphi=0$ or $0.5 \pi$ and squeezed degree increase accompanying with $m$ ascending under $m>0$ and $m$ descending under $m<0$. Under $m>0$, squeezing range increases when $m$ increases and then squeezing range almost doesn't change under $m<0$.

$<$ Figure 3>

In the fig 3, figures from (a) to (c) are drown under $m<0$. Figures from (e) to (f) are drown under $m>0$. Under $m>0, S^{c n}$ changes from positive to negative when $m$ increase. Under $m<0, S^{c n}$ always appears negative. Hence there is $c n$ squeezing effect at any $m<0$, and then there is $c n$ squeezing effect when $m$ is more than certain positive. Squeezing degree and squeezing range of $c n$ and $s n$ increase accompanying $|m|$ ascend. $S^{s n}$ always appears negative at any $m$.

\section{Conclusion}

In the paper, the squeeze properties of measurement phase operator are investigated in Superposition of Coherent State and Squeezed state. The below results are obtained: (1) there is a kind of squeezing effect of $C S$ in $Y_{l}$ under given parameters; (2) there is a kind of squeezing effect of $C S$ in $Y_{2}$ when $\varphi=0$ or $0.5 \pi$ and squeezed degree increase accompanying with $m$ ascending under $m>0$ and $m$ descending under $m<0$. Under $m>0$, squeezing range increases when $m$ increases and then squeezing range almost doesn't change under $m<0$; (3) there is $c n$ squeezing effect at any $m<0$, and then there is $c n$ squeezing effect when $m$ is more than certain positive. Squeezing degree and squeezing range of $c n$ and $s n$ increase accompanying $|m|$ ascend. $S^{s n}$ always appears negative at any $m$.

\section{References}

BARNETT S M, PEGG D T. (1986). Phase in quantum optics. Phys (A) Math Gen, 19: 3849.

LOUDON R, KNIGHT P L. (1987). Squeezed light. J Mod Opt, 34(6-7): 709-759.

LYNCH R. (1987). Phase fluctuations in a squeezed state using measured phase operators. J Opt Soc Am (B), 4 : 1723.

NATH R, KUMER P. (1991). Quasi-photon phase states. J Mod Opt, 38: 263.

PEGG D T, BARNETT S M. (1989). Phase properties of single-mode electromagnetic field. Phys Rev A, a39: 1665.

TU HSITEH, GONG CHANGDE. (1993). Properties of the measured phase operators in squeezed number states. J Mod Opt, 40: 57.

WALL D F. (1983). Squeezed States of Light. Nature, 306(10): 141-146. 


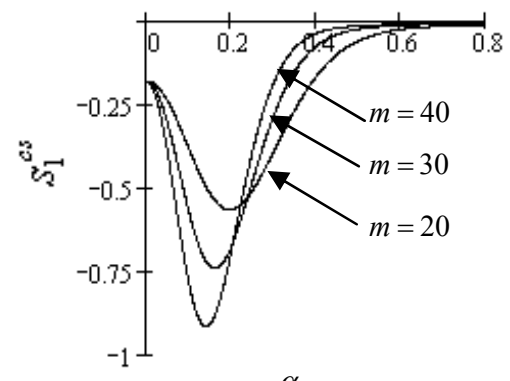

a. $r=0.6, \stackrel{\alpha}{=} 0$

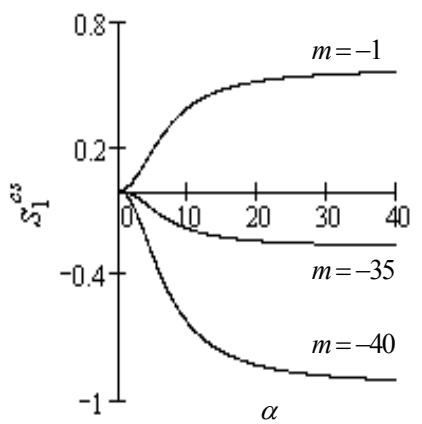

e. $r=2.6, \varphi=0$

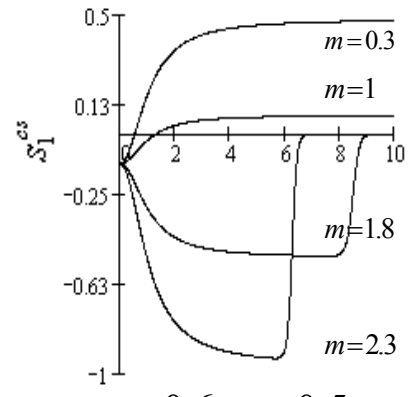

b. $r=0.6, \varphi=0.5 \pi$

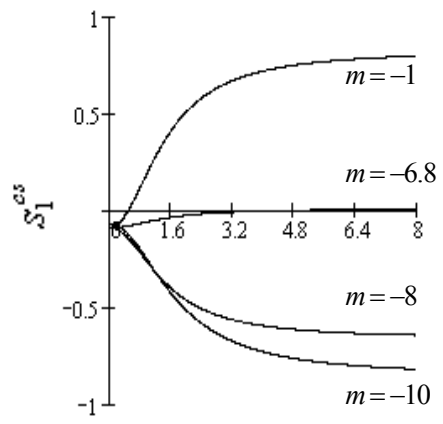

f. $r=1, \stackrel{\alpha}{\varphi}=0.5 \pi$

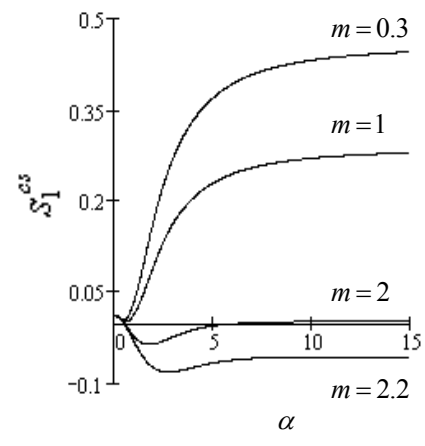

c. $r=1.5, \varphi=\pi$

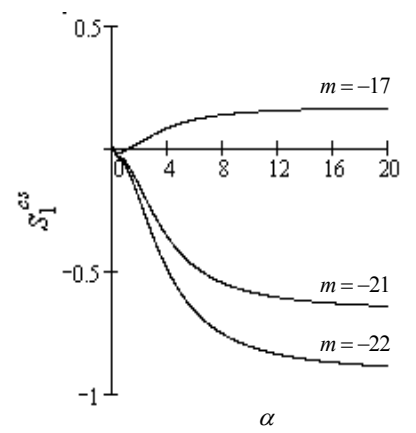

g. $r=2, \varphi=\pi$

Figure 1. The curve of degree of squeeze $S_{1}^{c s}$ varying with $\alpha, \varphi, r$ and $m$

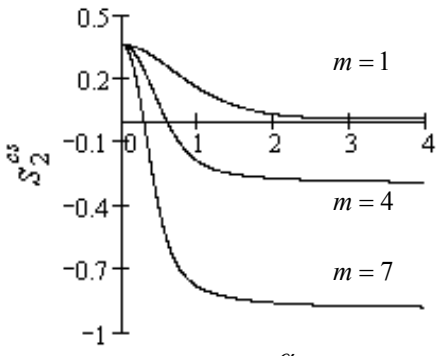

a. $r=0.6, \varphi=0$

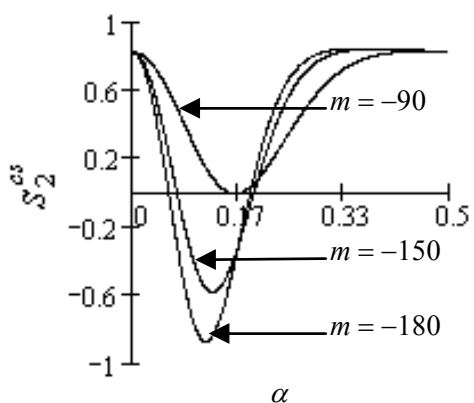

e. $r=1,5, \varphi=0$

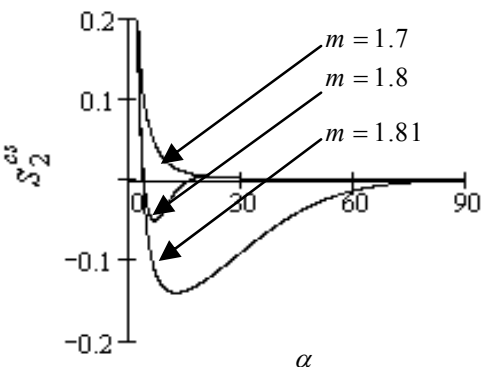

b. $r=1.2 .2, \varphi=0.5 \pi$

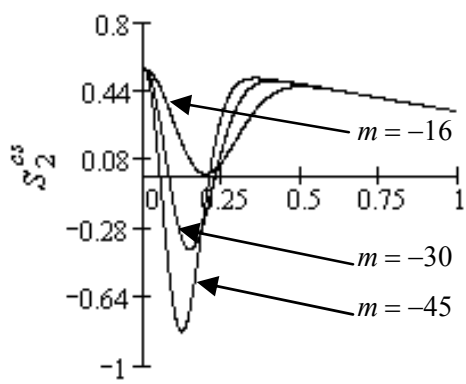

$\alpha$

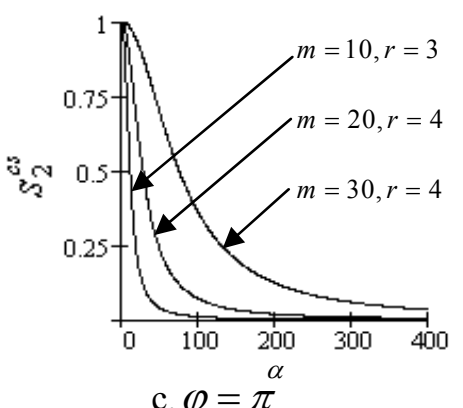

c. $\varphi=\pi$

Figure 2. The curve of degree of squeeze $S_{2}^{c s}$ varying with $\alpha, \varphi, \quad r$ and $m$ 


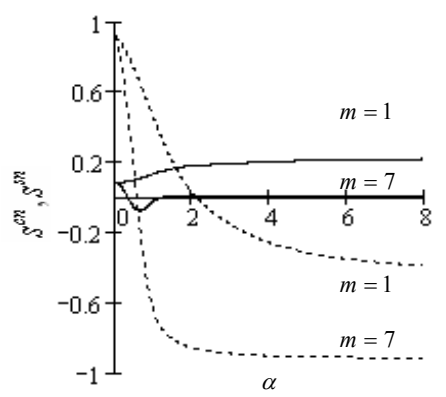

a. $r=1.2, \varphi=0$

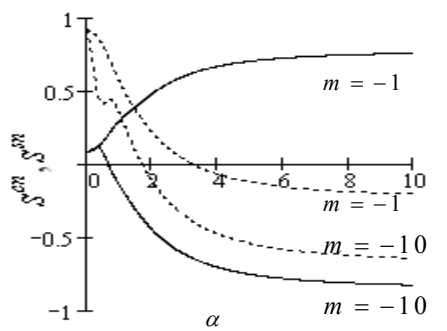

e. $r=1.2, \varphi=0$

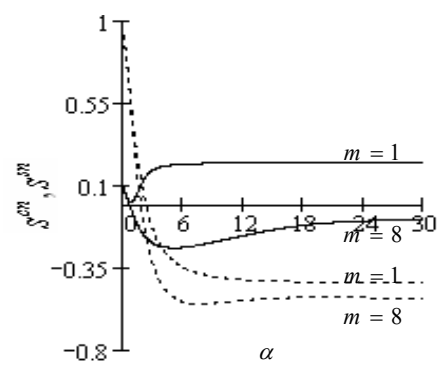

b. $r=1.2, \varphi=0.5 \pi$

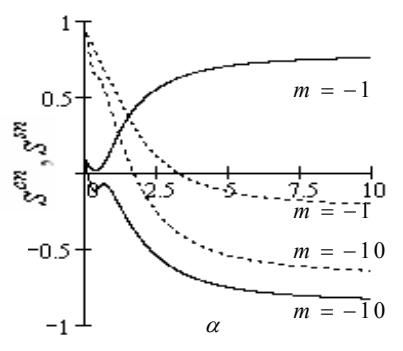

f. $r=1.2, \varphi=0.5 \pi$

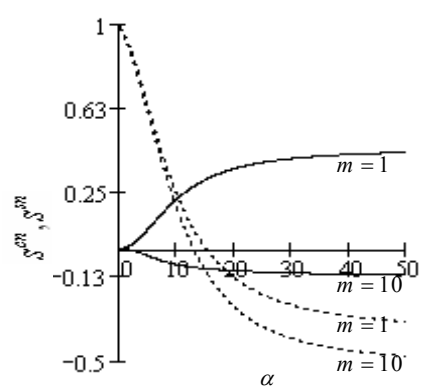

c. $r=3, \varphi=\pi$

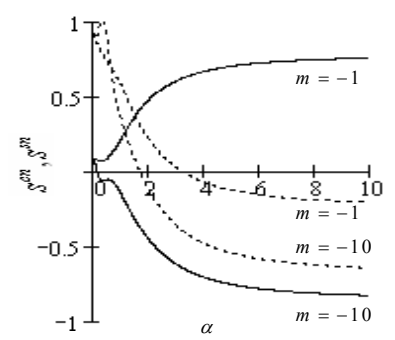

g. $r=1.2, \varphi=\pi$

Figure 3. The curve of degree of squeeze $S^{c n}$ and $S^{c n}$ varying with $\alpha, \varphi, r$ and $m$. Solid lines denote $S^{c n}$ and dot lines denote $S^{s n}$ 